\title{
Elaboração de muffins adicionados de espinafre e isentos de lactose e glúten
}

\author{
Thales Henrique Barreto Ferreira ${ }^{\mathrm{a} *} \mathbb{C}^{\circ}$, Ana Larissa Teodoro da Cunha ${ }^{\mathrm{b}}$, \\ Rita de Cássia Avellaneda Guimarães ${ }^{\mathrm{b}}$, Felicia Migumi Ito ${ }^{\mathrm{b}}$, Cláudia Leite Munhoz ${ }^{\mathrm{b}}$ ( \\ a Universidade Federal da Grande Dourados, Brasil \\ b Instituto Federal do Mato Grosso do Sul, Brasil \\ *Autor correspondente (thales_barreto25@hotmail.com)
}

\section{N F O}

\section{Keyworks}

Spinacea oleracea sensory acceptability characterization bread making

\section{Palavras-chaves \\ Spinacea oleracea aceitabilidade sensorial caracterização \\ minerais panificação}

\begin{abstract}
A B S T R A C T
Elaboration of added spinach muffins and lactose and gluten free.

Due to the concern with the maintenance of health, together with the increase in people with dietary restrictions, mainly allergies and intolerances, it was necessary to develop products that would serve this audience. In this sense, this study aims to prepare and characterize muffins added with spinach and free from lactose and gluten, in order to serve people with allergies and intolerances. Four muffin formulations were made: F1 (standard formulation: no spinach added, F2 (formulation with 25\% addition of spinach leaves), F3 (formulation with 50\% addition of spinach leaves), F4 (formulation with added $75 \%$ spinach leaves) keeping the same proportion for the other ingredients. Physical, chemical, microbiological, and sensory characterization were performed. The insertion of spinach reduced the diameter and thickness of the muffins with a variation of up to $8 \%$. The moisture content increased as the amount of spinach increased. The muffins were colored in black and green. All formulations showed microbiological stability. The sensorial acceptability was expressive, with scores higher than 6 , with samples being preferred to formulations F1 and F2. Thus, viability was observed in the production of muffins added with spinach and free from lactose and gluten. All formulations showed physical, chemical, and microbiological stability, being considered suitable for consumption.
\end{abstract}




\section{INTRODUÇÃO}

A crescente preocupação com a manutenção da saúde, juntamente com o aumento de pessoas com restrições alimentares, principalmente alergias e intolerâncias, estima-se que um entre 400 brasileiros possua alguma dessas restrições, assim se fazendo necessária uma mudança com relação à alimentação da população, necessitando de uma ingestão adequada de fibra alimentar, minerais e vitaminas (Mazzocchi et al., 2017; Ferreira et al., 2019).

As alergias alimentares são designadas por reações de hipersensibilidade; dentre essas, a doença celíaca é uma das principais alergias que atingem a população mundial, considerada uma reação imunológica ao glúten que causa uma inflamação grave no intestino; assim, restringindo e/ou impossibilitando o consumo de cereais, como cevada, centeio, aveia, trigo e malte. As intolerâncias alimentares podem provocar distúrbios gastrointestinais, cutâneos e respiratórios, destaca-se, principalmente a intolerância a lactose, dada pela incapacidade que o intestino tem de digerir e absorver a lactose, ou seja, o açúcar do leite, devido à deficiência da enzima denominada lactase (Rangel et al., 2016; Mansueto et al., 2015).

Devido à ausência do consumo destes alimentos vias alternativas se fazem necessárias, deste modo, necessita-se a criação de produtos e/ou preparos que contemplem a necessidade de nutrientes destes indivíduos, através da adição de alimentos ricos em vitaminas e minerais, como as hortaliças (Munhoz et al. 2018).

Dentre esses novos preparos e produtos, os muffins apresentam um grande potencial, pois sua formulação possibilita adição de novos ingredientes com apelos funcionais, ricos em nutrientes e compostos antioxidantes, além disso, destaca-se sua praticidade ao consumo e grande aceitabilidade sensorial (Ferreira et al., 2019).

Com relação aos novos ingredientes, o espinafre (Spinacea oleracea) é uma excelente opção de ingrediente alternativo nas formulações de muffins, pois, quando adicionados a dietas, o mesmo contribui com o aporte de vitaminas, proteína e minerais, especialmente cálcio e ferro, além de ser rico em compostos bioativos, como vitamina $\mathrm{C}$ e compostos fenólicos, assim, suprindo a necessidade de nutrientes desses indivíduos alérgicos e intolerantes (Santos e Boêno, 2016; Galla et al., 2017).

Neste sentido, este estudo teve como objetivo à elaboração e caracterização de muffins adicionados de espinafre e isentos de lactose e glúten, a fim de atender pessoas portadoras de alergias e intolerâncias.

\section{MATERIAL E MÉTODOS}

Para realização do estudo folhas de espinafre foram coletados numa propriedade rural localizada no município de Coxim-MS (latitude $18^{\circ} 28^{\prime} 57.9^{\prime \prime}$ 'S, longitude 54⒋'52.2' 'W). Os demais ingredientes utilizados nas formulações: farinha de arroz, cacau, açúcar tipo demerara, ovo, óleo, leite sem lactose e fermento químico em pó, foram adquiridos em comércio local. As folhas de espinafre foram selecionadas de acordo com sua integridade física, posteriormente, lavados em água corrente e sanitizadas por imersão em solução de hipoclorito de sódio a 200 ppm durante 15 minutos.

\section{Elaboração dos muffins}

Foram feitos testes preliminares variando as concentrações dos ingredientes, especialmente as folhas de espinafre, até as formulações obtidas.

Foram elaboradas quatro formulações de muffins: F1 (formulação padrão: sem adição de espinafre, F2 (formulação com adição de $25 \%$ de folhas de espinafre), F3 (formulação com adição de $50 \%$ de folhas de espinafre), F4 (formulação com adição de $75 \%$ de folhas de espinafre) mantendo a mesma proporção para os demais ingredientes (Tabela 1).

Tabela 1 - Formulação dos muffins adicionados de espinafre e isentos de lactose e glúten.

\begin{tabular}{l|c|c|c|c}
\hline \multicolumn{1}{c|}{ Ingredientes } & F1 & F2 & F3 & F4 \\
\hline Farinha de arroz $(\mathrm{g})$ & 155 & 155 & 155 & 155 \\
\hline Cacau $(\mathrm{g})$ & 30 & 30 & 30 & 30 \\
\hline Açúcar demerara $(\mathrm{g})$ & 114 & 114 & 114 & 115 \\
\hline Ovo $(\mathrm{g})$ & 100 & 100 & 100 & 100 \\
\hline Oléo $(\mathrm{mL})$ & 60 & 60 & 60 & 60 \\
\hline Fermento químico $(\mathrm{g})$ & 8 & 8 & 8 & 8 \\
\hline Leite sem lactose $(\mathrm{mL})$ & 150 & 150 & 150 & 150 \\
\hline Espinafre $(\mathrm{g})$ & $*$ & 117 & 235 & 330 \\
\hline
\end{tabular}

*não possui. 
A formulação de muffin padrão foi preparada de acordo com Ferreira et al. (2019) com algumas modificações: bateu-se o óleo $(60 \mathrm{~mL})$, os ovos $(100 \mathrm{~g})$ e o leite sem lactose $(150 \mathrm{~mL})$ por 5 minutos, em liquidificador na velocidade máxima; e adicionou-se as folhas de espinafre (apenas nas formulações F2, F3 e F4). Então, foram misturados aos ingredientes secos (farinha de arroz, açúcar demerara, cacau em pó e o fermento químico). A massa obtida foi enformada em formas metálicas revestidas com forminhas de papel e levada ao assamento em forno a temperatura $\left(180^{\circ} \mathrm{C}\right)$ por 15 min.

\section{Caracterização física e química}

$\mathrm{O}$ diâmetro e a espessura (mm) dos muffins foram determinados com auxílio de um paquímetro digital (Digimess), de acordo com os procedimentos descritos no método $10-50 \mathrm{D}$ da AACC (2000). A atividade de água (Aw) foi determinada por leitura direta em higrômetro digital (Aqualab, Decagon Devices Inc., EUA), previamente calibrado com soluções saturadas de sais de $\mathrm{NaCl}$ e $\mathrm{MgCl}_{2}$, respectivamente.

O conteúdo de umidade foi determinado pelo método gravimétrico em estuda a $70^{\circ} \mathrm{C}$ até obtenção de peso constante (AOAC, 1995). O conteúdo de resíduo mineral fixo foi calculado através da queima do excesso de açúcar com placa quente, seguida da incineração do mufla ( $C E$ $800 / S 4$ - CIENLAB) a $550^{\circ} \mathrm{C}$, até obtenção de cinzas claras e peso constante (AOAC, 1992).

1. Os parâmetros de cor dos muffins foram avaliados pelo método instrumental em cinco pontos de cada amostra, utilizando-se o colorímetro digital CR 400/410 (Konica Minolta), com determinação dos parâmetros $L^{*}$ (luminosidade), $a^{*}$ (variação de cor do verde ao vermelho), $b^{*}$ (variação de cor do azul ao amarelo), o parâmetro ${ }^{\circ} \mathrm{h}$ que define a tonalidade de cor e o parâmetro C que define a saturação da cor ou a cromaticidade foram calculados pelas Equações 1 e 2, respectivamente:

$$
\begin{aligned}
& h=\tan ^{-1}\left(\frac{a^{*}}{b^{*}}\right) \\
& C=\sqrt{\left(a^{*}\right)^{2}+\left(b^{*}\right)^{2}}
\end{aligned}
$$

A diferença total de cor $\left(\mathrm{AE}^{*}\right)$ entre a formulação padrão e aquelas que continham espinafre foi calculada utilizando a equação abaixo (Ferreira e Freitas, 2019):

$$
\Delta \mathrm{E} *=\sqrt{(\Delta L *)^{2}(\Delta a *)^{2}+(\Delta b *)^{2}}
$$

$\Delta \mathrm{E}=$ diferença de cor;
$\Delta L^{*}=\mathrm{Lc}$ (luminosidade do muffins padrão) $-\mathrm{Lt}$ (luminosidade do muffins teste);

$\Delta a^{*}=$ ac ( $a^{*}$ valores do muffins padrão) - at ( $a^{*}$ valores do muffins teste);

$\Delta b^{*}=\mathrm{bc}$ ( $b^{*}$ valores do muffins padrão) $-\mathrm{bt}\left(b^{*}\right.$ valores do muffins teste).

Os valores utilizados para determinar se a diferença total de cor foi visualmente identificada, foram os seguintes:

- $\Delta \mathrm{E}^{*}<1$ diferenças de cor não são identificadas pelo olho humano;

- $1<\Delta \mathrm{E}^{*}<3$ pequenas diferenças de cor podem ser identificadas pelo olho humano, dependendo da tonalidade;

- $\Delta \mathrm{E}^{*}>3$ as diferenças de cor são identificadas pelo olho humano.

\section{Caracterizaçãa Microbiológica}

Antes da análise sensorial, a fim de garantir a segurança microbiológica das amostras, foram realizados os teste de coliformes totais $\mathrm{e}$ termoresistenes e contagem de bolores e leveduras. Os procedimentos empregados no preparo das amostras foram realizados de acordo com a metodologia da American Public Health Association (APHA, 2001). A contagem de coliformes totais e termoresistentes foram realizadas pelo método do número mais provável (NMP) e de contagem de bolores e leveduras pelo método de unidades formadoras de colônia (UFC.g${ }^{1}$ ). As análises microbiológicas foram realizadas tendo como orientação a resolução $\mathrm{RDC}^{\circ} 12$, de 2 de janeiro de 2001 da ANVISA (Agência Nacional de Vigilância Sanitária) que aprova o regulamento técnico sobre padrões microbiológicos para alimentos (BRASIL, 2001).

\section{Análise sensorial}

A análise sensorial foi realizada no Laboratório de Análise Sensorial do Instituto Federal de Mato Grosso do Sul, campus Coxim. Os testes de aceitação foram realizados com 60 julgadores não treinados, que receberam quatro amostras codificadas com três dígitos. Juntamente, receberam uma ficha de avaliação, com teste de aceitabilidade das amostras por meio de uma escala hedônica de 9 pontos, que vai de 1 (desgostei muitíssimo) a 9 (gostei muitíssimo).

Os atributos avaliados na ficha foram aparência, cor, aroma, textura, sabor, sabor do espinafre, doçura e qualidade global. Cada julgador também indicou a intenção de compra para as amostras avaliadas (Dutcosky, 2013). Também foram avaliados, a frequência de consumo de muffins, amostra preferida, frequência de consumo de 
espinafre.

As amostras que obtiveram notas superior ou igual a 6 (gostei ligeiramente) foram consideradas aceitas. Além disso, foi calculado o índice de aceitação (IA) dos atributos sensoriais (Equação 3).

$$
I A(\%)=A \times \frac{100}{B}
$$

Em que A é a nota média obtida para o atributo e B é a nota máxima dada ao atributo.

A intenção de compra das amostras foram avaliadas pelos julgadores por meio de escala hedônica estruturada de 5 pontos variando entre certamente não compraria e certamente compraria.

\section{Análise estatística}

Todas as análises foram realizadas em triplicata e os resultados apresentados pela média $(n=3)$ e desvio padrão. As comparações entre os grupos foram submetidas à análise de variância (ANOVA) e quando significativas foram comparadas pelo teste de Tukey a 5\% de probabilidade de erro.

\section{RESULTADOS E DISCUSSÃO}

Na tabela 2 estão os resultados obtidos para a caracterização física e química dos muffins elaborados com folhas de espinafre e isentos de glúten e lactose. Para o diâmetro observou-se entre os tratamentos F1 e F4 diferença estatística entre si, embora os outros tratamentos não tenham se diferenciado, verificou-se redução no diâmetro à medida que se aumentou a quantidade de espinafre. A análise estatística mostrou que para a espessura, todas as formulações diferiram estatisticamente entre si, percebendo redução da espessura entre as formulações à medida que se aumentou o teor de espinafre. Comportamento similar foi observado por Ferreira et al. (2019) ao elaborar muffins com a adição de folhas de ora-pro-nobis. Essa diminuição tende a ocorrer, pois, com a inserção das folhas de espinafre nas formulações há uma dificuldade na liberação dos compostos voláteis durante a cocção dos muffins.

Tabela 2 - Caracterização física e química dos muffins adicionados de espinafre e isentos de lactose e glúten.

\begin{tabular}{l|l|l|l|l}
\hline \multirow{2}{*}{ Característica } & \multicolumn{3}{c}{ Formulac̃es } \\
\cline { 2 - 5 } & \multicolumn{1}{|c}{ F1 } & \multicolumn{1}{c}{ F2 } & \multicolumn{1}{c}{ F3 } \\
\hline Diâmetro $(\mathrm{cm})$ & $5,14 \pm 0,07 \mathrm{a}$ & $4,83 \pm 0,27 \mathrm{ab}$ & $4,75 \pm 0,16 \mathrm{ab}$ & $4,71 \pm 0,20 \mathrm{~b}$ \\
\hline Espessura $(\mathrm{cm})$ & $4,17 \pm 0,20 \mathrm{a}$ & $3,36 \pm 0,06 \mathrm{~b}$ & $2,10 \pm 0,26 \mathrm{c}$ & $2,06 \pm 0,17 \mathrm{c}$ \\
\hline Atividade de água $(\mathrm{adm})$ & $0,81 \pm 0,00 \mathrm{a}$ & $0,84 \pm 0,01 \mathrm{~b}$ & $0,85 \pm 0,01 \mathrm{~b}$ & $0,85 \pm 0,01 \mathrm{~b}$ \\
\hline Umidade $\left(\mathrm{g} .100 \mathrm{~g}^{-1}\right)$ & $29,15 \pm 0,13 \mathrm{a}$ & $39,51 \pm 0,05 \mathrm{~b}$ & $47,35 \pm 0,20 \mathrm{c}$ & $49,26 \pm 0,17 \mathrm{~d}$ \\
\hline Cinzas $\left(\mathrm{g} .100 \mathrm{~g}^{-1}\right)$ & $1,85 \pm 0,02 \mathrm{ab}$ & $2,76 \pm 0,06 \mathrm{a}, \mathrm{b}$ & $2,78 \pm 0,04 \mathrm{~b}$ & $2,90 \pm 0,05 \mathrm{a}$ \\
\hline
\end{tabular}

(F1: formulação padrão, sem adição de espinafre; F2: formulação com adição de $25 \%$ de folhas de espinafre; F3: formulação com adição de $50 \%$ de folhas de espinafre; F4: formulação com adição de $75 \%$ de folhas de espinafre). *Letras iguais na mesma linha não diferem entre si $(\mathrm{p}>0,05)$. Adm* Adimensional.

A atividade de água diferiu estaticamente da amostra F1 (sem adição de espinafre) das demais, essa diferenciação está relacionada à adição do espinafre nas formulações. Corroborando com esses resultados, comportamento similar foi observado para umidade, às amostras com maiores concentrações de espinafre apresentaram maiores conteúdos de umidade. Resultados semelhantes foram reportados por Santos e Boêno (2016) para muffins elaborados com e sem resíduo da polpa de graviola, a inserção de novos ingredientes na massa provoca o aumentou o teor de umidade.

A concentração da adição de espinafre foi considerada altamente significativa sobre o conteúdo residual fixo dos muffins, altos níveis foram observados na formulação $\mathrm{F} 2 \mathrm{com} 25 \%$ de espinafre $(2,76$ g. $100 \mathrm{~g}^{-1}$ ), seguido por F3 de 2,78 g. $100 \mathrm{~g}^{-1}$ e F4 de 2,90 g. $100 \mathrm{~g}^{-1}$ e o menor no controle F1 com 1,85 g. $100 \mathrm{~g}^{-1}$. Sing et. al. (2020) desenvolveram muffins ricos em ferro e obtiveram medias de 1,79 g. $100 \mathrm{~g}^{-1}$ de conteúdo mineral fixo, valor inferior ao deste estudo.
As médias da cor instrumental dos muffins adicionados de espinafre e isentos de lactose e glúten são apresentadas na Tabela 3. A cor dos produtos tendeu ao preto $\left(<L^{*}\right)$ em função da presença do cacau nas formulações, embora, observou-se que com a adição do espinafre houve um ligeiro aumento da coordenada $L^{*}$, observando diferença estatística da formulação padrão (F1) para as demais. A maior concentração (F4) apresentou menor coordenada $L^{*}$ de 18,82 . Valores semelhantes foram obtidos por Kuntz (2013), de 18,41 que desenvolveram muffins adicionados de inulina.

A coordenadas $b^{*}\left(-b^{*}\right.$ azul e $+b^{*}$ amarelo) não apresentou diferenças significativas entre as formulações. No entanto, com relação à coordenada $a^{*}$ ($a^{*}=$ verde e $+a^{*}$ vermelho) houve diminuição da coordenada (F1: 2,63 e F4: 1,05; respectivamente), isso ocorreu pela planta possuir cor característica de verde-escuro, a qual está associada à clorofila dos alimentos verdes, ela possui propriedades anticancerígenas, efeito desintoxicante das células e de inibição dos radicais livres (Rocha e Reed, 2014). 
Tabela 3 - Médias da cor instrumental dos muffins adicionados de espinafre e isentos de lactose e glúten.

\begin{tabular}{l|c|c|c|c}
\multirow{2}{*}{ Parâmetro } & \multicolumn{4}{|c}{ Formulações } \\
\cline { 2 - 5 } & F1 & F2 & F3 & F4 \\
\hline $\boldsymbol{L}^{*}$ & $11,17 \pm 0,62 \mathrm{a}$ & $13,86 \pm 1,04 \mathrm{~b}$ & $18,05 \pm 0,91 \mathrm{c}$ & $18,82 \pm 0,87 \mathrm{c}$ \\
\hline $\boldsymbol{a}^{*}$ & $2,63 \pm 0,56 \mathrm{a}$ & $1,80 \pm 0,33 \mathrm{ab}$ & $1,32 \pm 0,18 \mathrm{ab}$ & $1,05 \pm 0,15 \mathrm{~b}$ \\
\hline $\boldsymbol{b}^{*}$ & $4,16 \pm 0,61 \mathrm{a}$ & $3,91 \pm 0,22 \mathrm{a}$ & $3,67 \pm 0,41 \mathrm{a}$ & $4,11 \pm 0,43 \mathrm{a}$ \\
\hline $\boldsymbol{C}^{*}$ & $4,93 \pm 0,81 \mathrm{a}$ & $4,31 \pm 0,27 \mathrm{a}$ & $3,90 \pm 0,44 \mathrm{a}$ & $4,24 \pm 0,45 \mathrm{a}$ \\
\hline${ }^{\circ} \mathbf{h}$ & $57,87 \pm 1,61 \mathrm{a}$ & $64,00 \pm 3,34 \mathrm{~b}$ & $70,11 \pm 1,71 \mathrm{c}$ & $75,67 \pm 0,78 \mathrm{~d}$ \\
\hline$\Delta \mathrm{E}$ & $*$ & 2,82 & 6,98 & 7,81 \\
\hline
\end{tabular}

(F1: formulação padrão, sem adição de espinafre; F2: formulação com adição de $25 \%$ de folhas de espinafre; F3: formulação com adição de $50 \%$ de folhas de espinafre; F4: formulação com adição de $75 \%$ de folhas de espinafre). *Letras iguais na mesma linha não diferem entre si $(\mathrm{p}>0,05)$.

Segundo Kirca et. al. (2007), o valor de croma (C*) descreve a saturação ou intensidade da cor; portanto, associasse que valores mais altos de $\mathrm{C}^{*}$ apresentam características de cores mais intensas; consequentemente, eles são mais atraentes e mais bem aceitos pelos consumidores. Os valores de $\mathrm{C}^{*}$ (croma) não se diferiram estatisticamente, o que indica que eles têm saturações semelhantes; diferentemente do ângulo Hue $\left({ }^{\circ} \mathrm{h}\right)$ que diferiu entre todas as formulações. De acordo com Kuntz et. al. (2013) amostras com $\Delta \mathrm{E}$ maiores de 3 as diferenças de cor da amostra padrão ao teste são identificadas pelo olho humano, neste sentindo, somente a amostra F2 não consegue ser percebida a variação de cor de forma visível.

Em relação aos resultados das análises microbiológicas para as diferentes formulações de muffins adicionados de espinafre, não houve contagens de coliformes totais, tal pouco como termotolerantes; a contagem de fungos e leveduras também não foi identificada, o que lhes confere conformidade com os regulamentos (Brasil, 2001) e segurança no consumo.

A tabela 4 apresenta as médias de aceitabilidade sensorial das formulações de muffins adicionados de espinafre e isentos de lactose e glúten. As médias para todos os atributos foram superiores a 6,0 (gostei ligeiramente). Ductkosky (2013) apresenta que para uma amostra ser considerada como aceita deve possuir médias superiores a 6,0; desta maneira, pode-se observar que todas as amostras foram consideradas aceitas pelos julgadores.

As médias para qualidade global foram estatisticamente menores para as formulações F3 e F4, assim como para o atributo aparência, indicando que o aumento do percentual de espinafre diminuiu a aceitabilidade da qualidade global, o mesmo comportamento foi percebido para a aparência. A formulação F3 apresentou também menor nota de sabor quando comparado as demais formulações. Com relação aos outros atributos, não houve diferença estatística entre eles. Os escores do atributo "sabor do espinafre" chama atenção, pois, observase que houve diferença estatística entre as amostras, muitos consumidores de maneira geral associam a presença do espinafre em formulações como de "sabor desagradável", contudo, pode-se afirmar que os muffins não apresentaram sabor residual da hortaliça, favorecendo assim seu consumo.

Tabela 4 - Médias do teste de aceitabilidade sensorial de muffins adicionados de espinafre e isentos de lactose e glúten.

\begin{tabular}{|c|c|c|c|c|}
\hline \multirow{2}{*}{ Atributo } & \multicolumn{4}{|c|}{ Formulações } \\
\hline & F1 & F2 & F3 & F4 \\
\hline Aparência & $7,68 \mathrm{a}$ & $7,35 \mathrm{~b}$ & $7,18 \mathrm{~b}$ & $7,12 \mathrm{~b}$ \\
\hline Cor & $7,54 \mathrm{a}$ & $7,42 \mathrm{a}$ & $7,28 \mathrm{a}$ & $7,40 \mathrm{a}$ \\
\hline Aroma & $7,31 \mathrm{a}$ & $7,18 \mathrm{a}$ & $7,08 \mathrm{a}$ & $7,20 \mathrm{a}$ \\
\hline Textura & $7,35 \mathrm{a}$ & $7,58 \mathrm{a}$ & $6,95 \mathrm{a}$ & $7,29 \mathrm{a}$ \\
\hline Sabor & $7,22 \mathrm{ab}$ & $7,54 \mathrm{a}$ & $6,71 b$ & $7,02 \mathrm{ab}$ \\
\hline Sabor do espinafre & $6,42 \mathrm{a}$ & $6,86 \mathrm{a}$ & $6,40 \mathrm{a}$ & $6,80 \mathrm{a}$ \\
\hline Doçura & $7,22 \mathrm{a}$ & $7,31 \mathrm{a}$ & $6,77 \mathrm{a}$ & $7,03 \mathrm{a}$ \\
\hline Qualidade global & $7,40 \mathrm{ab}$ & $7,74 \mathrm{a}$ & $7,11 \mathrm{~b}$ & $7,17 \mathrm{~b}$ \\
\hline
\end{tabular}

(F1: formulação padrão, sem adição de espinafre; F2: formulação com adição de 25\% de folhas de espinafre; F3: formulação com adição de $50 \%$ de folhas de espinafre; F4: formulação com adição de $75 \%$ de folhas de espinafre). *Letras iguais na mesma linha não diferem entre si $(\mathrm{p}>0,05)$.

Na tabela 5 encontram-se os Índices de Aceitabilidade (IA) para os atributos avaliados nos muffins e na Figura 2, encontra-se o gráfico da preferência. De acordo com o IA, as amostras de 
muffins padrão (F1) e com $25 \%$ de espinafre (F2) apresentaram maiores índices de aceitabilidade, o que corrobora com as amostras preferidas escolhidas pelos julgadores (Figura 2). As formulações padrão e com $25 \%$ de espinafre foram às preferidas pelos julgadores. Augusto et al. (2017) tiveram índice de aceitabilidade de $82,4 \%$ para cupcake elaborados com farinhas de talos de espinafre e couve, o que mostra a boa aceitabilidade de produtos enriquecidos com vegetais, podendo ser uma alternativa de produto a ser oferecido.

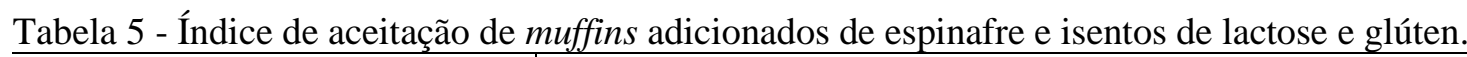

\begin{tabular}{l|c|c|c|c}
\hline \multirow{2}{*}{ Atributos } & \multicolumn{4}{c}{ Formulações } \\
\cline { 2 - 5 } & F1 & F2 & F3 & F4 \\
\hline Aparência & 85,3 & 81,7 & 79,8 & 79,1 \\
\hline Cor & 83,8 & 82,4 & 80,9 & 82,2 \\
\hline Aroma & 81,2 & 79,8 & 78,7 & 80,0 \\
\hline Textura & 81,7 & 84,2 & 77,2 & 81,0 \\
\hline Sabor & 80,2 & 83,7 & 74,6 & 78,0 \\
\hline Sabor do espinafre & 71,3 & 76,2 & 71,1 & 75,6 \\
\hline Doçura & 80,2 & 81,2 & 75,2 & 78,1 \\
\hline Qualidade global & 82,2 & 86,0 & 79,0 & 79,7 \\
\hline
\end{tabular}

(F1: formulação padrão, sem adição de espinafre; F2: formulação com adição de 25\% de folhas de espinafre; F3: formulação com adição de $50 \%$ de folhas de espinafre; F4: formulação com adição de $75 \%$ de folhas de espinafre). *Letras iguais na mesma linha não diferem entre si $(\mathrm{p}>0,05)$. Dados expressos em $\%$.

Na análise de preferência (Figura 1), as formulações padrão (F1) e com $25 \%$ de espinafre (F2) foram às preferidas pelos julgadores, cerca de $60 \%$ apontaram com sua amostra preferida. A formulação com adição de $50 \%$ de espinafre (F3) foi a menos preferida pelos julgadores $(15 \%)$.

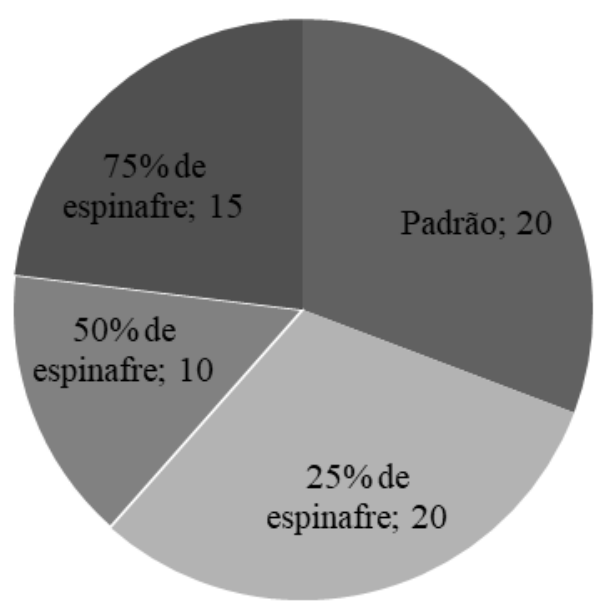

Figura 1 - Gráfico de preferência dos muffins adicionados de espinafre e isentos de lactose e glúten.

A intenção de compra para os muffins (Figura 2), independente da formulação, demonstra perspectivas positivas, pois, grande maioria dos julgadores apontaram que comprariam o produto, caso ele estivesse disponível. 


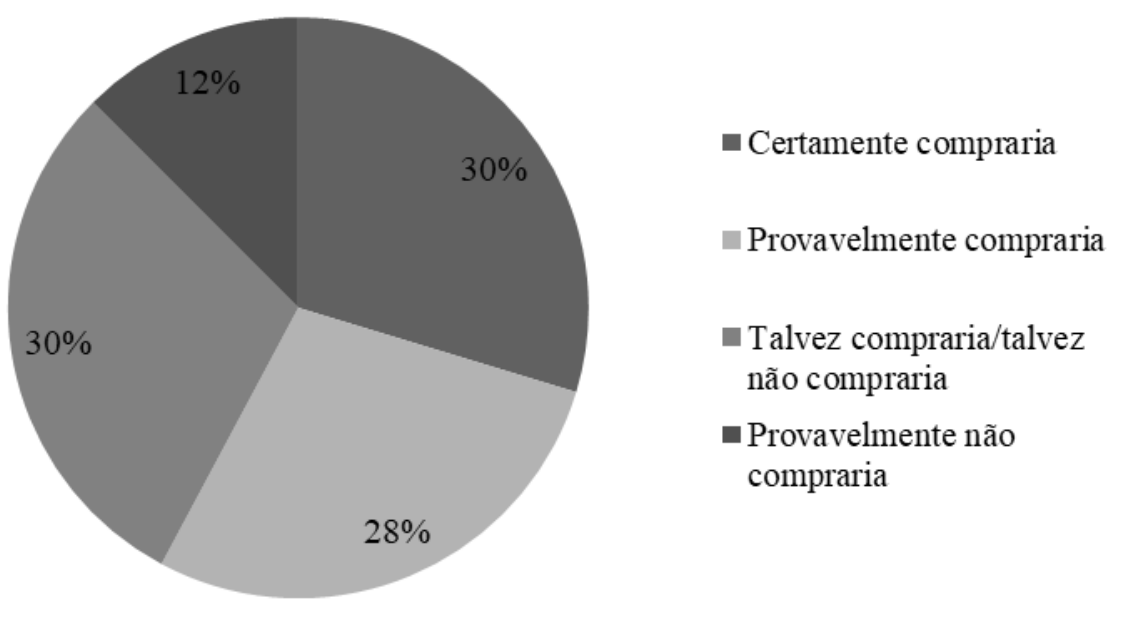

Figura 2 - Intenção de compra dos muffins adicionados de espinafre e isentos de lactose e glúten.

A frequência de consumo de muffins (Figura 3) foi de $49 \%$ para o consumo de três vezes na semana, o que indica que muffins são produtos presente para quase metade dos julgadores e de amplo consumo. Com relação à frequência de consumo de espinafre $44 \%$ apontaram que consomem a hortaliça

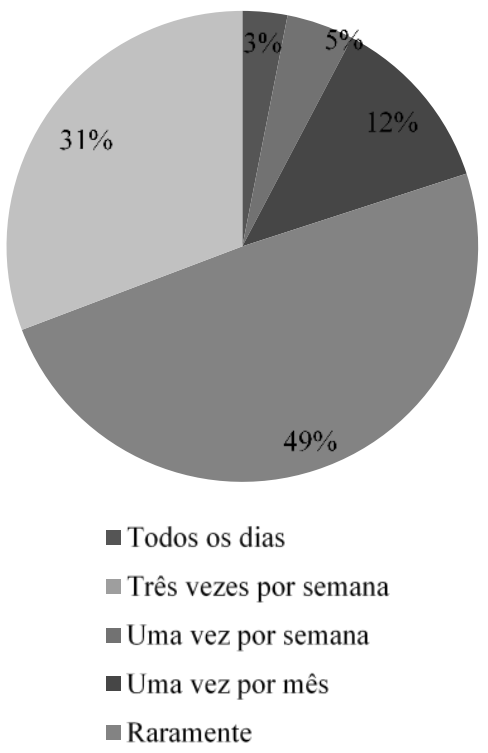

(a) semanalmente. Ambos os resultados projetam a possibilidade de consumo e comercialização de muffins adicionados de espinafre, corroborando com valores da aceitabilidade sensorial, novas perspectivas partem desses resultados obtidos.

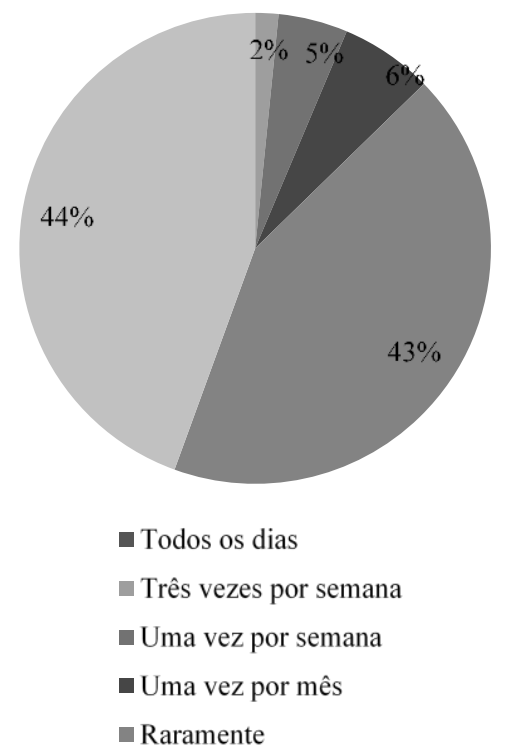

(b)

Figura 3 - (a) Frequência de consumo de muffins pelos julgadores; (b) Frequência de consumo de espinafre pelos julgadores.

\section{CONCLUSÕES}

Foi observado viabilidade na produção de muffins adicionados de espinafre e isentos de lactose e glúten. Todas as formulações apresentaram estabilidade física, química e microbiológica, sendo considerados aptos ao consumo.

As formulações apresentaram boa aceitação sensorial, sendo preferida a formulação F2 (com adição de $25 \%$ de espinafre).

A intenção de compra foi de $58 \%$ entre certamente compraria e provavelmente compraria o produto. 


\section{AGRADECIMENTOS}

Ao Conselho Nacional de Desenvolvimento Científico e Tecnológico (CNPq), a Fundação de Apoio ao Desenvolvimento do Ensino, Ciência e Tecnologia do Estado de Mato Grosso do Sul (FUNDECT) e ao Instituto Federal do Mato Grosso do Sul (IFMS).

\section{REFERÊNCIAS BIBLIOGRÁFICAS}

AACC. American Association of Cereal Chemists. Approved methods of the AACC. 9.ed. St. Paul: AACC, 1995. 1200 p.

American Public Health Association - APHA. Compendium of methods for the microbiological examination of foods. Washington, DC, 2001. $676 \mathrm{p}$.

AOAC. Association Of Oficial Analytical Chemistral. Official methods of analysis of the association of official analytical chemistry. 11th ed, AOAC, Arlington. 1992.

AOAC. Association Of Oficial Analytical Chemistral. Official methods of analysis of the association of official analytical chemistry. 16th ed, AOAC, Arlington. 1995.

Augusto G, Zanlourensi CB, Chiconatto P, Schmitt P. Aceitação de cupcakes com farinha de talos de couve manteiga e farinha de talos de espinafre por escolares do município de Prudentópolis-PR. Revista Brasileira de Obesidade, $\mathrm{Nu}-$ trição e Emagrecimento, v. 68, p.731-737, 2017.

BRASIL. Ministério da Saúde. Agência Nacional de Vigilância Sanitária. Resolução RDC No 12 de 02 de janeiro de 2001. Aprova o regulamento Técnico sobre Padrões Microbiológicos para Alimentos. Diário Oficial da União, Brasília, 10 de janeiro de 2001.

Dutcosky, S. D. Análise sensorial de alimentos. Curitiba: Champagnat. 2013

Ferreira THB, Basaglia RR, Cunha TMP, Argandona EJS. Muffins de beterraba adicionados de ora-pro-nobis: Caracterização física. Anais do III Sinatex, v.3, p.179-184, 2019.

Ferreira THB, Freitas MLF. Production, physical, chemical and sensory evaluation of dried banana (Musa Cavendish). Emirates Journal of Food and Agriculture, v.31, p.102-108, 2019.

Galla NR, Pamidighantam PR, Karakala B, Rudrayya M, Akula GS. Nutritional, textural and sensory quality of biscuits supplemented with spinach (Spinacia oleracea L.). International Journal of Gastronomy and Food Science, v.7, p. 20-26, 2017.

Kirca A, Özkan M, Cemeroğlu B. Storage stability of strawberry jam color enhanced with black carrot juice concentrate. Journal Food Procesess and Preservation, v.31, p.531-545, 2007.

KUNTZ MGF. Efeitos da inulina nas propriedades físicas, químicas, de textura e aceitabilidade no desenvolvimento de muffin destinado a consumidores em idade escolar. 2013. 150 f. Tese (Doutorado em Ciência de Alimentos)Programa de Pós-Graduação em Ciência e Tecnologia de Alimentos, Universidade Federal de Santa Catarina, Florianópolis, 2013.
Mansueto P, D'alcamo A, Seidita A, Carroccio A. Food allergy in irritable bowel syndrome: The case of non-celiac wheat sensitivity. World Journal of Gastroenterology, v.21, p.7089-7109, 2015. https://doi.org/10.3748/wjg.v21.i23.7089

Mazzocchi A, Venter C, Maslin K, Agostoni C. The Role of Nutritional Aspects in Food Allergy: Prevention and Management. Nutrients, v.9, p.850-857, 2017.

Munhoz CL, Ferreira THB, Florencio EMC, Souza LH. Desenvolvimento de Barra de Cereais de Castanha de Baru Enriquecidas com Prebióticos. Cadernos de Agroecologia, v. 13, p. 1-7, 2018.

Rangel AHN, Vendas DC, Urbana AS, Galvão Júnior JGB, Andrade Neto JC, Macêdo CS. Lactose intolerance and cow's milk protein allergy. Food Science and Technology, v.36 p.179-187, 2016. https://doi.org/10.1590/1678457X.0019

Rocha DS, Reed E. Pigmentos Naturais em Alimentos e sua Importância para Saúde. Estudos, v.41, n.1, p. 76-85. 2014.

Santos JR, Boêno JA. Muffins isentos de glúten e lactose desenvolvidos com resíduo de polpa de graviola. Revista de Agricultura Neotropical, v.3, p.42-51, 2016. 